\title{
Cerebellar Theta and Beta Noninvasive Stimulation Rhythms Differentially Influence Episodic Memory versus Semantic Prediction
}

\author{
${ }^{\circledR}$ Shruti Dave, ${ }^{\circledR}$ Stephen VanHaerents, and ${ }^{\circledR}$ Joel L. Voss \\ Department of Medical Social Sciences, Ken and Ruth Davee Department of Neurology, Department of Psychiatry and Behavioral Sciences, and \\ Interdepartmental Neuroscience Program, Feinberg School of Medicine, Northwestern University, Chicago, Illinois 60611
}

The human cerebellum is thought to interact with distributed brain networks to support cognitive abilities such as episodic memory and semantic prediction. Hippocampal and fronto-temporo-parietal networks that respectively support episodic memory versus semantic prediction have been associated with distinct endogenous oscillatory activity frequency bands: theta $(\sim 3-8 \mathrm{~Hz})$ versus beta $(\sim 13-30 \mathrm{~Hz})$ respectively. We sought to test whether it is possible to toggle cerebellar participation in episodic memory versus semantic prediction by noninvasively stimulating with theta versus beta rhythmic transcranial magnetic stimulation. In human subjects of both sexes, cerebellar theta stimulation improved episodic memory encoding but did not influence neural signals of semantic prediction, whereas beta stimulation of the same cerebellar location increased neural signals of semantic prediction but did not influence episodic memory encoding. This constitutes evidence for double dissociation of cerebellar contributions to semantic prediction versus episodic memory based on stimulation rhythm, supporting the hypothesis that the cerebellum can be biased to support these distinct cognitive abilities at the command of network-specific rhythmic activity.

Key words: beta frequency; EEG; episodic memory; language processing; theta frequency; transcranial magnetic stimulation

Significance Statement

The cerebellum interacts with several distinct large-scale brain networks for cognitive function, but the factors governing selectivity of such interactions for particular functions are not fully understood. We tested the hypothesis that cerebellar contributions to cognition are guided by neural oscillations with function-specific frequency bands. We demonstrated that matching noninvasive stimulation to network-specific frequencies selectively enhanced episodic memory versus semantic prediction. These findings suggest that cerebellar contributions to cognitive networks are selected based on corresponding activity rhythms and could be used to develop cerebellar stimulation interventions for specific neurocognitive impairments.

Received Mar. 12, 2020; revised May 21, 2020; accepted June 29, 2020.

Author contributions: S.D., S.V., and J.L.V. designed research; S.D. performed research; S.D. and J.L.V. analyzed data; S.D. and J.L.V. wrote the paper.

The authors declare no competing financial interests.

This research was supported by Grants R01-MH106512 and F32-MH118718-01 from the National Institutes of Health. Neuroimaging was performed at the Northwestern University Center for Translational Imaging, which is supported by the Northwestern University Department of Radiology. This research was supported, in part, through the computational resources and staff contributions provided for Quest, the high-performance computing facility at Northwestern University, which is jointly supported by the Office of the Provost, the Office for Research, and Northwestern University Information Technology. We thank M. Penckofer, S.E. Lurie, M.M. Gunlogson, B.E. Durr, J.E. Kragel, and M.S. Hermiller for assistance with the experiment. The content is solely the responsibility of the authors and does not necessarily represent the official view of the National Institutes of Health.

Correspondence should be addressed to Shruti Dave at shruti.dave@northwestern.edu.

https://doi.org/10.1523/JNEUROSCI.0595-20.2020

Copyright $\odot 2020$ the authors

\section{Introduction}

The cerebellum contributes to a number of cognitive abilities, including episodic memory, language, decision-making, motor control, and others (Desmond and Fiez, 1998; Schmahmann, 2019). Many reports have particularly implicated lobules VI and VII, and especially Crus I and II in cognition (Stoodley, 2012). Portions of these cerebellar regions show resting-state fMRI connectivity and task-based coactivation with most major distributed cognitive brain networks (Buckner et al., 2011; King et al., 2019). For example, Crus I is a functionally heterogeneous lobule of posterolateral cerebellar cortex (Buckner et al., 2011; Stoodley, 2012) that interacts with the hippocampal network that supports episodic memory (Fliessbach et al., 2007; Krienen and Buckner, 2009; Buckner et al., 2011) and with the fronto-temporo-parietal network that supports language functions such as semantic prediction (De Smet et al., 2013; King et al., 2019), alongside other functional networks (Buckner et al., 2011; King et al., 2019). The 


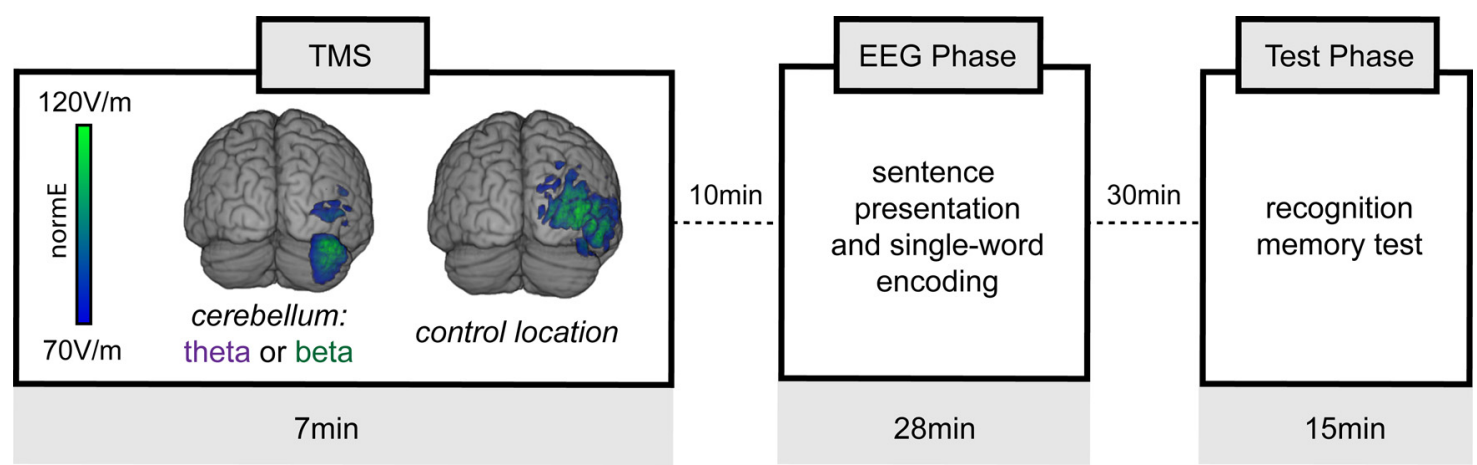

Figure 1. Experiment design. Each subject participated in three separate sessions. For each session, one of the following three TMS conditions was delivered: cerebellar theta (theta-burst), cerebellar beta $(20 \mathrm{~Hz})$, or control-location stimulation (either theta or beta). The induced electrical field (normE) is illustrated for a sample participant. Following stimulation on each session, subjects performed a mixed sentence reading and single-word encoding task while scalp EEG was recorded. This portion of the task occurred during the period when the effects of stimulation on neural function were likely maximal. Recognition memory for words studied during the EEG phase was tested after a delay, such that retrieval occurred after the effects of stimulation had decayed (Materials and Methods).

goal of the current experiment was to evaluate whether such interactions are functional by testing whether noninvasive stimulation targeting cerebellar Crus I can differentially impact hallmark behavioral and neural correlates of the hippocampal network that supports episodic memory versus the fronto-temporo-parietal network that supports semantic prediction.

The stimulation approach in this experiment was motivated by previous evidence that networks supporting episodic memory versus semantic prediction are associated with distinct frequencies of oscillatory activity. Theta-band $(\sim 3-8 \mathrm{~Hz})$ interregional synchronicity of hippocampal network locations, including the cerebellum, has been associated with memory encoding and retrieval (Anderson et al., 2010; Lega et al., 2012; Herweg et al., 2020), which is consistent with the dominant theta activity pattern of the hippocampus (Larson et al., 1986; Zhang and Jacobs, 2015; Bohbot et al., 2017). In contrast, beta-band $(\sim 13-30 \mathrm{~Hz})$ activity is prominent among fronto-temporo-parietal network locations, including the cerebellum (Bastiaansen and Hagoort, 2006; Weiss and Mueller, 2012), particularly during language tasks in which sentence context supports generating expectations or predictions for specific words (i.e., semantic prediction during reading; Engel et al., 2001; Lewis and Bastiaansen, 2015; Molinaro et al., 2016). We therefore hypothesized that transcranial magnetic stimulation applied to Crus I using theta versus beta rhythmic patterns could bias its participation in episodic memory versus semantic prediction.

Several previous findings support the premise that noninvasive stimulation applied at theta versus beta frequencies may differentially affect the networks of interest. For instance, theta-burst transcranial magnetic stimulation (TMS) applied to a cortical location of the hippocampal network enhanced episodic memory performance, whereas beta-frequency TMS of the same location did not affect memory (Hermiller et al., 2019). In contrast, beta-frequency TMS of a cortical location of the fronto-temporo-parietal network enhanced picture naming, whereas $1 \mathrm{~Hz}$ TMS did not alter picture naming (Mottaghy et al., 1999; Sparing et al., 2001). Importantly, these previous experiments used different stimulation locations within either network and did not assess the frequency selectivity of stimulation effects on memory-related versus language-related functions. Therefore, it remains unclear whether a single location such as Crus I can be biased to contribute to distinct functions by applying theta versus beta noninvasive stimulation.

The experiment we conducted to test this possibility follows the logic of the "double dissociation" (Teuber, 1955), whereby we tested whether two distinct cognitive outcome measures are differentially modulated by two distinct stimulation interventions. We applied TMS targeting cerebellar Crus I using either theta or beta rhythms (vs a control location in occipital cortex) and measured the impact of stimulation using gold standard assessments of episodic memory versus semantic prediction. We hypothesized that cerebellar stimulation rhythm would differentially impact these outcomes, with relative increases in episodic memory performance following cerebellar theta rhythmic stimulation and relative increases in neural signals of semantic prediction following beta rhythmic stimulation.

\section{Materials and Methods}

\section{Overview}

Participants performed cognitive tasks assessing highly canonical measures of semantic prediction and episodic memory encoding (Fig. 1). Stimulation effects on semantic prediction were assessed via brain electrical activity measured during reading comprehension trials designed to elicit the event-related brain potential (ERP) N400 signal (Kutas and Federmeier, 2011). Stimulation effects on episodic memory encoding were assessed by measuring subsequent high-confidence performance on a delayed recognition memory test (Shepard, 1967; Eichenbaum et al., 2007) administered after the period during which stimulation typically impacts neural function (Di Lazzaro et al., 2005; Huang and Kandel, 2005), and by examining ERPs recorded during encoding (Rugg and Yonelinas, 2003). As is typical for experiments following the doubledissociation logic, these measures of cognitive function differed fundamentally in format. Nonetheless, these distinct functional outcomes are advantageous because they are well validated probes of semantic prediction versus episodic memory (Yonelinas, 2002; Rugg and Yonelinas, 2003; Kutas and Federmeier, 2011), thus permitting strong inferences regarding any differential influence of cerebellar theta versus beta stimulation on cognition.

We also applied the same stimulation rhythms to a control location of right lateral occipital cortex, as this location is unrelated to episodic memory and language networks and therefore served as an active stimulation control (Fig. 1). We hypothesized that cerebellar beta stimulation would influence the N400 signal of semantic prediction but have relatively little impact on memory encoding, whereas cerebellar theta stimulation would influence memory encoding but have relatively little impact on the N400 signal. This hypothesis that a differential impact of stimulation rhythm on distinct semantic prediction versus episodic memory assessments follows the same logic as the aforementioned double-dissociation experiments, and would thereby provide strong evidence that lateral cerebellum (including Crus I) is rhythm selective in its support of episodic memory versus semantic prediction.

\section{Participants}

Subjects $(N=25)$ had normal or corrected-to-normal vision and passed MRI and TMS safety screenings (Rossi et al., 2009) supervised by S.V. 
Subjects had no known history of psychiatric or neurologic disorder. All provided written informed consent to a protocol approved by the Institutional Review Board at Northwestern University and were paid for their participation. Data from one subject were excluded from analyses because of excessive artifacts in EEG recordings (see below). Reported analyses thus include 24 adults (16 women; average age: 25.3 years; age range: $18-37$ years).

\section{Experiment design}

Before the three experiment sessions, each subject completed a structural MRI scan to provide anatomic localization for TMS targeting. The experiment used a within-subjects design across three sessions performed on nonconsecutive days (average intersession interval: $6 \mathrm{~d}$; range: 2-26d). During each session, one of three stimulation conditions was delivered immediately before the EEG phase, which was then followed by the test phase (Fig. 1). The EEG phase comprised $\sim 28$ min during which sentence and single-word materials were presented while EEG was recorded (see below). Stimulation order across sessions was counterbalanced across subjects. Visual stimuli were counterbalanced across stimulation conditions and session orders. EEG caps were affixed to subjects and prepared before stimulation, such that presentation of experimental materials began $10 \mathrm{~min}$ after the final stimulation pulse for each session. The EEG phase was followed by a $30 \mathrm{~min}$ delayed word recognition task test phase, during which EEG was not recorded.

\section{TMS protocol}

During each session, subjects first received one of three TMS conditions: cerebellar theta $(5 \mathrm{~Hz}$ delivery of $50 \mathrm{~Hz}$ bursts), cerebellar beta $(20 \mathrm{~Hz})$, or location control stimulation. Location control stimulation was delivered at the right lateral occipital cortex and was counterbalanced between theta and beta patterned stimulation across subjects.

MRI data were collected using a Siemens 3 T Prisma whole-body scanner with a 64-channel head-neck coil located in the Northwestern University Center for Translational Imaging. Structural images were acquired using a T1-weighted MPRAGE sequence (176 frames; TE: $1.69 \mathrm{~ms}$; TR: $2170 \mathrm{~ms}$; TI: $1160 \mathrm{~ms}$; flip angle: $7^{\circ}$; voxel resolution: $1.0 \times$ $1.0 \times 1.0 \mathrm{~mm}$; 1-mm-thick sagittal slices; $256 \times 256 \mathrm{~mm}$ FOV; scan duration: $5.1 \mathrm{~min}$ ). Structural MRI data were preprocessed using AFNI software (version AFNI_19.3.04; Cox, 1996). Scans were skull stripped (3dSkullStrip) and warped into Talairach-Tournoux (TT) stereotactic space using the TT_N27 atlas (auto_tlrc). Lateral cerebellar and occipital control-location stimulation sites were selected using a priori coordinates and are listed in MNI space for convenience [cerebellum MNI: 41, $-79,-39$ (Halko et al., 2014); occipital MNI: 47, $-78,-13$ defined based on lack of membership in memory and language cortical network parcellations (Yeo et al., 2011; Shain et al., 2020)]. Each stimulation location was adjusted per subject to fall on the nearest adjacent brain surface (mean distance from default target: $1.7 \mathrm{~mm}$ for cerebellum; $3.6 \mathrm{~mm}$ for occipital) and then transformed back into subject-specific original MRI space for anatomically guided TMS. The average distance between cerebellar and control-location stimulation targets in original space was 29.6 $\mathrm{mm}(\mathrm{SD}=1.7 \mathrm{~mm})$. TMS delivery at the control location feels very similar to stimulation at the lateral cerebellum because of its relative proximity. Furthermore, visual cortical contributions to sentence reading and single-word reading trials are likely very similar, and therefore the occipital cortex serves as an adequate control stimulation location.

Beta and theta stimulation both comprised 600 bipolar pulses applied at individualized intensity. For beta stimulation, pulses were delivered in 1540 -pulse $20 \mathrm{~Hz}$ trains ( $2 \mathrm{~s}$ on, $28 \mathrm{~s}$ off; $\sim 7 \mathrm{~min}$ total duration; i.e., 20 $\mathrm{Hz}$ repetitive TMS). For theta stimulation, $50 \mathrm{~Hz}$ pulse triplets were delivered every $200 \mathrm{~ms}(5 \mathrm{~Hz})$ for $40 \mathrm{~s}$ continuously (i.e., continuous theta-burst stimulation). To better match the subjective duration of stimulation conditions, theta stimulation was preceded by $\sim 6.5 \mathrm{~min}$ of beta stimulation delivered at a very low, subthreshold intensity $(10 \%$ of resting motor threshold; i.e., the sound, but not the intensity, of beta stimulation). Each subject received theta stimulation and beta stimulation of the cerebellar target across different sessions. For the control-location session, half of the subjects received theta stimulation and half received beta stimulation. All stimulation conditions were counterbalanced across session order and were matched across sessions for stimulation intensity (calibrated per subject). Subjects were informed that stimulation pattern and location and thus sensation would vary among sessions but were unaware of location- or stimulation-specific study hypotheses.

We delivered TMS via a MagPro X100 system with a Cool-B65 coil (MagVenture) using frameless stereotactic guidance (Localite). Maximum stimulation intensity was calibrated to $80 \%$ of the resting motor threshold identified for the right abductor pollicis brevis before the experimental sessions. Intensity was adjusted for comfort in 13 subjects, resulting in an average stimulation intensity of $78.7 \%$ resting motor threshold (42.8\% stimulator maximum output; range: $32-55 \%)$. For subjects with adjusted intensity, the same intensity was used for all stimulation conditions.

TMS was delivered to optimize induced electric field orientation, directed mediorostrally for the cerebellum and mediolaterally for the occipital cortex (Janssen et al., 2015). Finite element modeling using subject-specific MRI was used to calculate induced electrical field following cerebellar and location control stimulation (Fig. 1, representative subject) implemented in SimNIBS version 3.0 (Thielscher et al., 2015).

\section{Combined semantic prediction and episodic memory task}

EEG phase. Eighty sentences and 64 single words were presented during each session. Subjects were instructed to read each sentence for comprehension, which was tested by manual response to true/false statements following 16 trials. Subjects were additionally informed that they would be tested on word memory. At each session, the subject performed a brief ( $\sim 5 \mathrm{~min})$ practice including the delayed memory test.

Stimuli included 480 sentence pairs with 240 unique sentence-final critical words (similar to materials presented in the study by Dave et al., 2018). The following two factors varied between sentences in each pair: cloze, or the likelihood a sentence would end in a specific sentence-final word; and contextual constraint, or the highest probability a sentence would end in any final word. One sentence of each pair was strongly constrained toward a highly expected (i.e., high cloze) ending (average cloze: $90 \%$; range: $80-100 \%$; e.g., "Mark hates raw fish, so he refuses to eat sushi"). These endings are referred to as "high predictability." The second sentence in each pair was weakly constrained (average constraint: $21.3 \%$; range: $6-30 \%$ ) and ended with a low cloze, or unlikely to be predicted, final word (average cloze: $1.1 \%$, range: $0-4 \%$; e.g., "It is common to use your hands to eat sushi"). These endings are referred to as "low predictability." Averages for cloze and constraint were obtained from 50 additional adults (average age: 36.4 years; range: 22-68 years) recruited and compensated via Amazon Mechanical Turk.

Sentence length was approximately matched across sentence pairs generated for the comprehension task (i.e., within 2 words for each pair; average length: 9 words; range: 6-11 words). Critical words had a mean SUBTLEX-US frequency count of 1215 , a mean length of 5.4 letters, and a mean concreteness rating of 3.9 (Brysbaert et al., 2014). Frequency, word length, and concreteness ratings of sentence-final words were matched across the 80 sentences selected for each session.

We created a list of 192 separate words for single-word trial presentation. For the single words, the mean frequency count was 1182, mean word length was 5.4 letters, and a mean concreteness rating was 3.8. Sixty-four words were presented during each session, matched on frequency, length, and concreteness across sessions.

During the EEG phase of each session, each subject read 40 high-predictability and 40 low-predictability sentences that were randomly intermixed, such that each critical word and its corresponding sentence was presented once per subject. The sentences were counterbalanced across two experimental lists so that each target word appeared equally often in high- and low-predictability sentences. To test sentence comprehension, subjects were asked to evaluate true/false statements on $20 \%$ of trials (average accuracy: $92.3 \%$, range: $75-100 \%$ ), indicating that subjects both understood sentence content and were paying adequate attention while reading. Sixty-four single-word trials were presented during each session, randomly intermixed with sentence trials.

Before each trial, a cue (e.g., SENTENCE or WORD) was displayed for $2000 \mathrm{~ms}$ to indicate upcoming trial type to subjects. Cue presentation 
was followed by a $4000 \mathrm{~ms}$ display of a central fixation cross. Sentences were presented via rapid serial visual presentation with a stimulus-onset asynchrony of $600 \mathrm{~ms}$ ( $300 \mathrm{~ms} /$ word). Single words were similarly presented for $300 \mathrm{~ms}$ following two buffer items ("XXXXXX") used to standardize reading preparedness between sentence and single-word trials. A blank screen was presented for $1200 \mathrm{~ms}$ after sentence-final target words and single-word trials, followed by a $4000 \mathrm{~ms}$ display of a central fixation cross between trials.

Continuous EEG was recorded from 27 active $\mathrm{Ag} / \mathrm{AgCl}$ electrodes mounted in an elastic cap (actiCAP, Brain Vision). Electrodes were not positioned at areas where TMS was delivered to facilitate cerebellar and occipital stimulation. To monitor saccades and blink activity, four additional electrodes were placed below and on the outer canthi of the left and right eyes. All electrode impedances were kept below $10 \mathrm{k} \Omega$. The EEG signal was amplified online (bandwidth: 20,000 Hz) and was digitized continuously at a sampling rate of $1000 \mathrm{~Hz}$ along with stimulusonset codes used for subsequent averaging. All channels were referenced to the right mastoid and rereferenced offline to the average of right and left mastoids. Data were collected via Pycorder (Python) and processed via MATLAB (MathWorks) using ERPlab plugins (Lopez-Calderon and Luck, 2014).

Delayed recognition test phase. Following the EEG phase for each session, memory was tested for the words encountered during the EEG phase (64 sentence-final words, excluding those that were paired with comprehension questions during study, and 64 single words). These studied words were randomly intermixed with an equal number of novel lure words matched on length, frequency, and concreteness with studied words. Subjects attempted to discriminate studied from lure words using a 4-point confidence scale (confident old, guess old, guess new, and confident new). Words were presented individually alongside the confidence scale, and subjects were given up to $5 \mathrm{~s}$ to respond, with the recognition response cueing delivery of the next word. The primary episodic memory outcome measure was memory performance for single words, given the uncertainty about the cognitive processes reflected by recognition of sentence-ending words.

\section{Statistical analysis}

Analyses were performed in MATLAB (version R2017b) and R (version R-3.6.2). For ERP analyses, continuous EEG data were epoched in $1200 \mathrm{~ms}$ intervals starting $200 \mathrm{~ms}$ before the onset of sentence-final words and individually presented words. Independent component analysis (ICA) was performed (Lopez-Calderon and Luck, 2014) to isolate and remove blink and saccade components. Components that included only frontal and ocular channels were removed (mean $=3.8$ components, $\mathrm{SD}=1.5$ components removed). Following ICA, single-trial waveforms were screened for artifactual contamination (i.e., muscle or nonblink eye movement) at an absolute voltage threshold of $100 \mu \mathrm{V}$. An average of $3.1 \%$ of sentence final-word trials and $5.9 \%$ of single-word trials were excluded from further analysis (no significant differences across stimulation or day order in the number of excluded trials: $p$ values $>0.08$ ).

Primary analyses examined the effects of stimulation type (stimulation: cerebellar beta, cerebellar theta, location control) on outcome measures. EEG activity during sentence-final word trials were binned by offline sentence cloze probability (cloze: high cloze, low cloze) and subsequently averaged to create ERPs. Main analyses of sentence-final words focused on the N400 signal (Kutas and Federmeier, 2011; Dave et al., 2018), which has typically been defined as a negative-going difference between high- and low-cloze trials maximal over central and posterior electrode sites (CP1, CP2, CZ, C3, C4, Pz, P3, P4, O1) 300-500 ms after word onset. We calculated the mean ERP amplitude of 300-500 ms averaged for this set of electrodes for both cloze conditions and calculated the N400 signal by subtracting amplitudes for low cloze from those for high cloze.

We performed additional analyses on four other ERP components associated with language processing (described in Results). We measured the P150 (Holcomb and Grainger, 2006; measured for 100-200 ms following word onset) and the posterior syntactic P600 (Leckey and Federmeier, 2020; measured 600-900 ms after word onset) at central and posterior electrodes (CP1, CP2, Cz, C3, C4, Pz, P3, P4, O1). We also calculate mean ERP amplitudes over a set of frontocentral electrodes (FP1, FP2, FC1, FC2, Fz, FC5, FC6) to assess the following two additional language-related effects: the N200/N250 (Holcomb and Grainger, 2006) measured for 150-300 ms following word onset) and the frontal semantic P600/PNP (Van Petten and Luka, 2012) measured for 600-900 $\mathrm{ms}$ following word onset).

Behavioral analyses for delayed recognition of individually studied words focused on discrimination sensitivity $\left(\mathrm{d}^{\prime}\right)$, a normalized metric of discrimination of studied from novel lure words (Yonelinas, 2002). Response rates for each response type (old/new) and confidence level (confident/guess) and stimulus type (studied/foil) are listed for each condition in Extended Data Figure 4-1. As indicated in the Results, only confident responses were accurate. Therefore, we analyzed the effect of stimulation on $\mathrm{d}^{\prime}$ calculated only for confident responses. We additionally examined ERP correlates of word encoding following similar procedures as for the N400 analysis. We calculated mean ERP amplitude for central and posterior electrodes where the parietal memory effect is typically maximal (CP1, CP2, Cz, C3, C4, Pz, P3, P4, O1) 650-750 ms after word onset for all studied single-word trials.

For ERP and recognition analyses, we performed 1-by-3 repeatedmeasures (RM) ANOVAs with stimulation condition as the withinsubject factor (control location, cerebellar beta, cerebellar theta). RM-ANOVAs were followed by pairwise stimulation condition comparisons corrected using Tukey's method.

\section{Results}

\section{Frequency-dependent stimulation effects on N400 correlates of semantic prediction}

We first tested whether cerebellar stimulation frequency influenced the N400 ERP component, which is robustly modulated by the expectancy or predictability of words (Fig. 2; Kutas and Federmeier, 2011). The N400 is typically maximal for centroposterior electrodes $\sim 300-500 \mathrm{~ms}$ following the onset of less predictable compared with highly predictable sentence-final words. As expected, subjects demonstrated typical effects of predictability on N400 amplitudes (i.e., ERP amplitude differences between high-predictability and low-predictability words measured at centro-posterior electrodes from 300 to $500 \mathrm{~ms}$ ) for each of the three stimulation conditions (Fig. 2; high-predictability vs lowpredictability amplitude for location control stimulation: $t_{(23)}=$ 4.32, $p=0.0003, d=0.83$; cerebellar-beta stimulation: $t_{(23)}=6.72$, $p<0.0001, d=1.33$; cerebellar-theta stimulation: $t_{(23)}=5.43, p<$ $0.0001, d=1.16$; all paired $t$ tests).

As hypothesized, stimulation condition significantly influenced the effect of predictability on N400 amplitudes (Fig. 3A; main effect: $\left.F_{(2,46)}=4.83, p=0.01, \eta_{p}^{2}=0.21\right)$. Cerebellar beta stimulation enhanced the N400 effect amplitude relative to control-location stimulation $\left(t_{(46)}=3.11, p=0.009, d=0.67\right)$. In contrast, cerebellar theta stimulation did not significantly influence the N400 predictability effect amplitude relative to control-location stimulation $\left(t_{(46)}=1.47, p=0.24\right)$ or relative to cerebellar beta stimulation $\left(t_{(46)}=1.64, p=0.32\right)$.

As the N400 predictability effect was calculated as the difference between high-predictability and low-predictability words (Kutas and Federmeier, 2011; Kuperberg and Jaeger, 2016), we performed post hoc analyses to better understand how cerebellar beta stimulation increased the N400 effect relative to controllocation stimulation (Fig. $3 B$ ). Beta stimulation numerically but nonsignificantly increased N400 amplitudes to high predictability words relative to control-location stimulation $\left(t_{(23)}=1.27\right.$, $p=0.29)$. N400 amplitudes to low-predictability words were significantly lower following cerebellar beta stimulation than control-location stimulation $\left(t_{(23)}=4.84, p=0.04, d=0.17\right)$. Thus, the influence of beta stimulation on the effects of predictability 
A
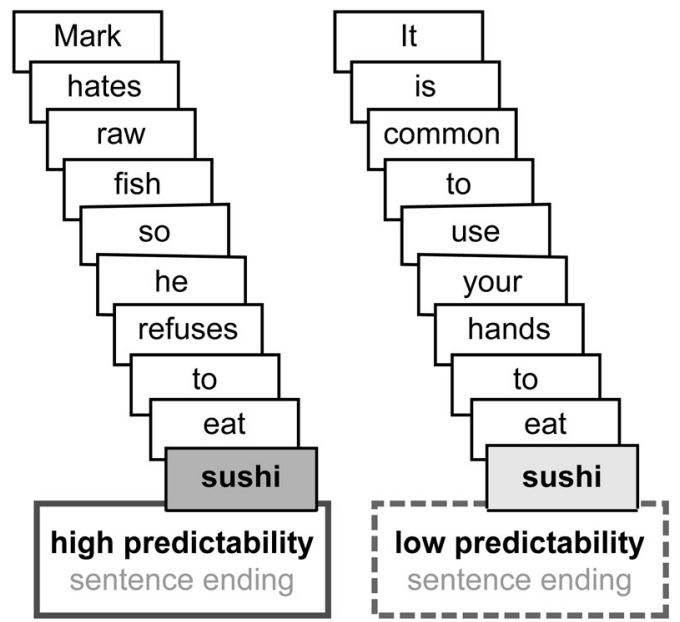

C

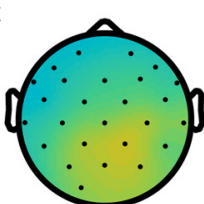

controllocation

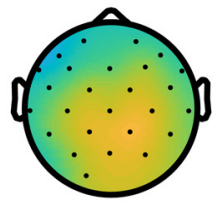

cerebellar theta

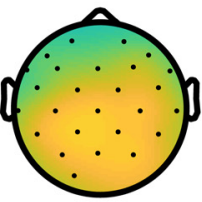

cerebellar

beta

$-4 \mu \mathrm{V}$

$+4 \mu \mathrm{V}$
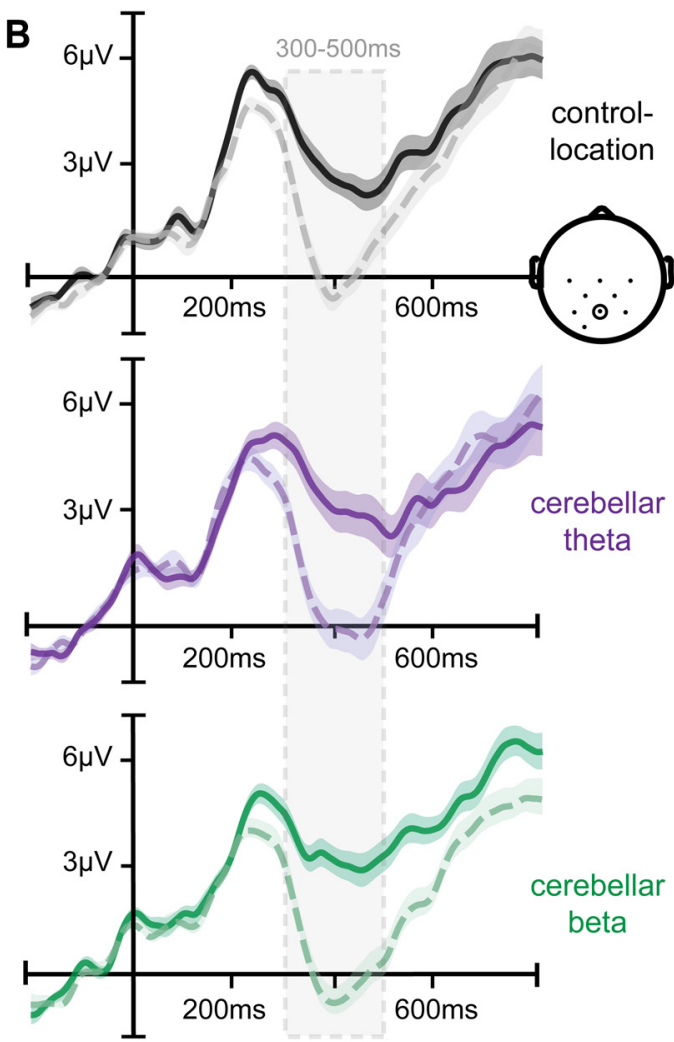

Figure 2. Typical ERP signals of semantic prediction for each stimulation condition. $\boldsymbol{A}$, Example sentences for high versus low predictability sentence-final words. $\boldsymbol{B}$, Average ERPs to high-predictability (solid) and low-predictability (dashed) words, plotted for a representative centroposterior electrode (Pz) for all three stimulation conditions. C, Scalp topography of the N400 effect (i.e., difference between high-predictability and low-predictability words) for each stimulation condition shows a greater and more widespread N400 distribution following cerebellar beta stimulation relative to control-location stimulation. Shading on ERP waveforms indicates the SD of the group mean.
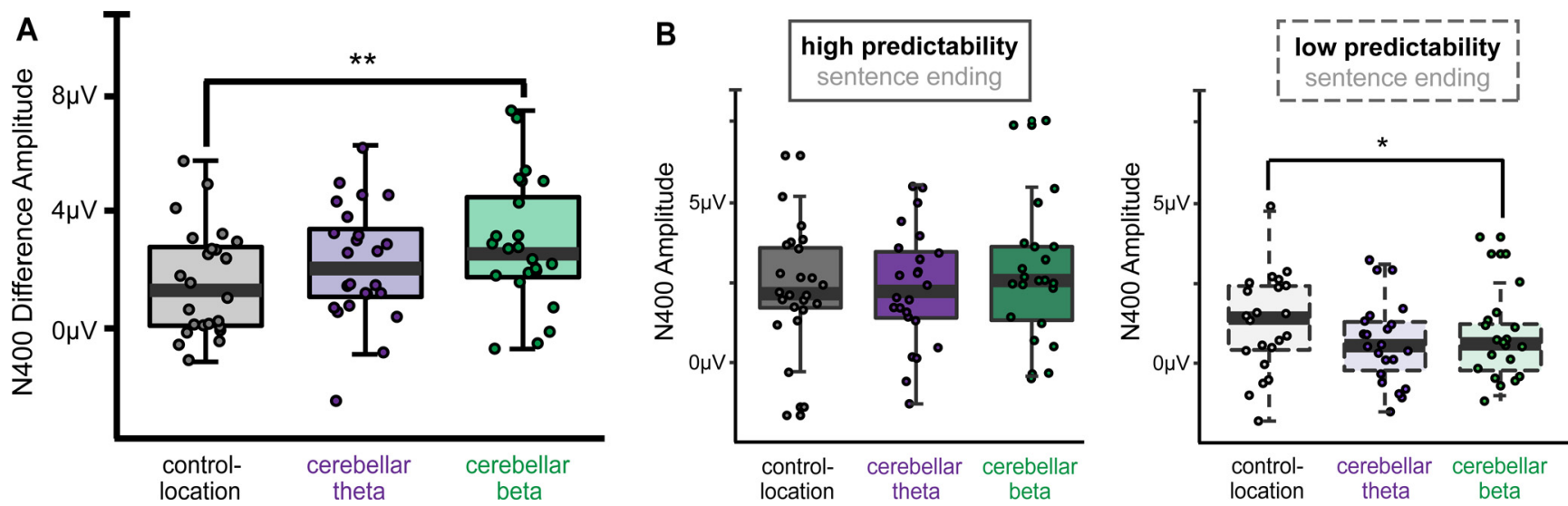

Figure 3. Cerebellar beta stimulation increased ERP signal of semantic prediction. $A$, Average $N 400$ high-predictability minus low-predictability difference amplitudes ( $300-500 \mathrm{~ms}$ ) for each stimulation condition. $\boldsymbol{B}$, Average $\mathrm{N} 400$ amplitudes for high-predictability and low-predictability words. Whiskers indicate first and third quartiles. Dots indicate values for individual subjects. ${ }^{* *} p<0.01 ;{ }^{*} p<0.05$.

on N400 reflected a combination of small increases in N400 amplitude to high-predictability words and decreases in N400 to low-predictability words, suggesting that word processing was more strongly influenced by the predictability of the sentence context (Kuperberg and Jaeger, 2016) following cerebellar beta stimulation.

Control analyses indicated that the effect of predictability on N400 difference amplitudes (i.e., difference between high-predictability and low-predictability words) did not vary by session order regardless of stimulation condition (all pairwise $p$ values $>0.91$ ). Thus, session-to-session practice or ordering effects did not significantly alter N400 amplitudes and therefore did not contribute to the influence of stimulation on N400 amplitudes. Furthermore, there was no significant difference in the effect of predictability on N400 amplitudes for subjects receiving beta stimulation of the control location versus subjects receiving theta stimulation of the control location $\left(t_{(23)}=1.21\right.$, $p=0.24)$, indicating that rhythm-specific stimulation effects were unique to the cerebellum.

To evaluate cognitive specificity, we examined the effects of stimulation on N400 amplitudes measured to words presented individually, as these words lacked sentence context and 

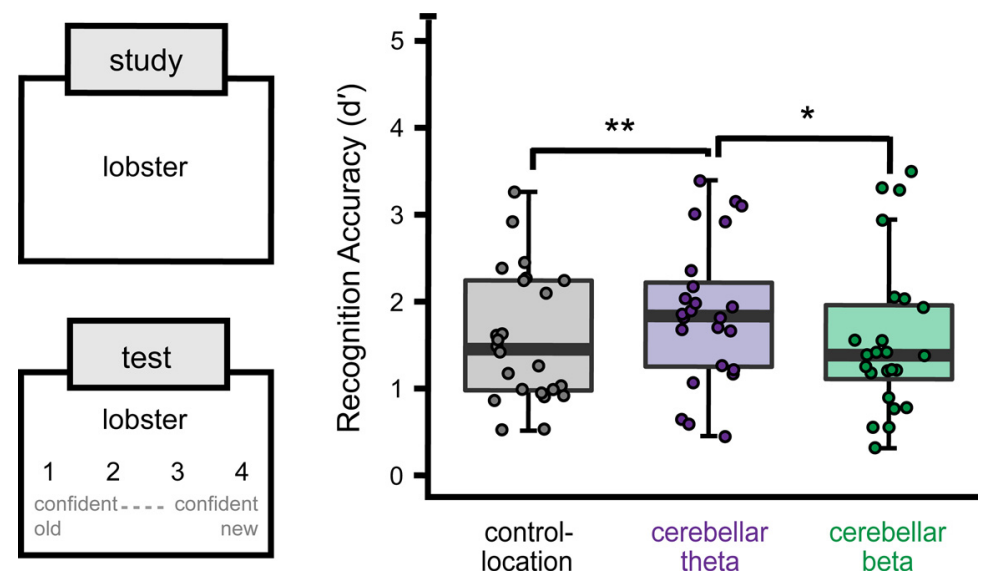

Figure 4. Cerebellar theta stimulation enhances episodic memory. Words were studied individually during the EEG phase, and a recognition memory test was administered after a delay. Subjects attempted to discriminate studied words from novel foils and simultaneously rated confidence. Recognition discrimination accuracy for confident responses $\left(d^{\prime}\right)$ varied by stimulation condition, as shown via box-and-whisker plots with whiskers marking the first and third quartiles (for supplementary details, see Extended Data Fig. 4-1). Dots indicate values for individual subjects. ${ }^{*} p<0.05 ;{ }^{* *} p<0.01$.

therefore should not have involved the same levels of language processing related to contextual predictability. N400 amplitudes to individually presented words did not vary significantly by stimulation condition (main effect: $F_{(2,46)}=1.09, p=0.35, \eta_{p}^{2}=0.05$; these ERPs are shown below: recognition memory ERPs). Therefore, the effects of cerebellar beta stimulation on N400 amplitudes were specific to sentence/contextual processing.

To further evaluate the selectivity of the predicted effect of beta stimulation on the N400, we performed exploratory control analyses of other ERP components often measured during language tasks. We examined the frontal post-N400 positivity (i.e., semantic P600, or PNP) associated with costs of incorrect predictions (Van Petten and Luka, 2012), the posterior post-N400 positivity (i.e., syntactic $\mathrm{P} 600$ ) associated with grammatical processing difficulties or animacy violations (Leckey and Federmeier, 2020), the frontal N200/N250 associated with early visual word form processing (Holcomb and Grainger, 2006), and the P150 component, which is sensitive to letter or word form reflecting early orthographic processing (Holcomb and Grainger, 2006). There were no ERP amplitude differences between high-predictability and low-predictability words for any of the three stimulation conditions for frontal post-N400 positivity, for posterior post-N400 positivity, or for P150 amplitude (all pairwise $p$ values $>0.08$ ). N200/N250 amplitudes differed by word predictability following cerebellar beta stimulation $\left(t_{(23)}=\right.$ 3.09, $p=0.01, d=0.13)$ and control-location stimulation $\left(t_{(23)}=\right.$ 2.23, $p=0.04, d=0.14$ ), with a marginal difference for cerebellar theta stimulation $\left(t_{(23)}=1.92, p=0.07\right)$. However, contrary to the effects observed on the N400, stimulation condition did not modulate the effect of word predictability (high-predictability vs low-predictability amplitude difference) for the $\mathrm{N} 200 / \mathrm{N} 250$ component $\left(F_{(2,46)}=0.33, p=0.72\right)$. These control analyses indicate that these nonhypothesized ERP components were not sensitive to the task for any stimulation condition and did not show a significant differential effect of stimulation rhythm.

\section{Rhythm-dependent stimulation effects on episodic memory encoding}

We next evaluated the effects of stimulation on episodic memory encoding of words presented individually, which were studied intermixed with sentence trials. These words were studied during the EEG phase within 45 min of stimulation delivery (i.e., within the period when the stimulation parameters have been found to impact neural activity and cognitive function; Rossi et al., 2009; Hoogendam et al., 2010; Thut and Pascual-Leone, 2010). The recognition memory test was administered following a delay (Fig. 1), after the expected duration of impact for the current stimulation conditions. During the recognition test, subjects discriminated studied words from novel foils and simultaneously rated memory confidence (Jacoby, 1983; Yonelinas, 1999). For all stimulation conditions on average, subjects made more confident "old" responses to studied words than novel foils $\left(t_{(71)}=15.17, p<0.001, d=2.52 ; \mathrm{d}^{\prime}\right.$ mean $=1.65$, $\mathrm{d}^{\prime} \mathrm{SD}=0.82$; Extended Data Fig. 4-1), indicating successful discrimination. However, discrimination was at chance when subjects made guess responses (similar "old" endorsement rates for studied words and novel foils; $t_{(71)}=0.60$, $p=0.55 ; \quad d^{\prime} \quad$ mean $\left.=0.02, \quad d^{\prime} \quad S D=0.42\right)$. As intended, only confident recognition trials were highly accurate and likely reflect the recollection component of episodic memory that depends on the hippocampal network (Yonelinas, 2002; Diana et al., 2007; Eichenbaum et al., 2007). We therefore tested the effects of cerebellar stimulation on the accuracy of confident responses.

Confident recognition discrimination accuracy was influenced by stimulation condition (Fig. 4; main effect: $F_{(2,46)}=5.61$, $\left.p=0.01, \eta_{p}^{2}=0.24\right)$. Cerebellar theta stimulation enhanced accuracy relative to cerebellar beta stimulation $\left(t_{(46)}=2.87, p=0.02\right.$, $d=0.35)$ and relative to control-location stimulation $\left(t_{(46)}=2.93\right.$, $p=0.009, d=0.38)$. In contrast, cerebellar beta stimulation did not significantly influence accuracy relative to control-location stimulation $\left(t_{(46)}=0.06, p=0.998\right)$. The beneficial effects of cerebellar theta stimulation on discrimination accuracy were related to increased hit rates relative to control-location stimulation $\left(t_{(23)}=2.09, p=0.05, d=0.20\right)$ as well as reduced false alarm rates relative to control-location stimulation $\left(t_{(23)}=2.26, p=0.03\right.$, $d=0.27)$ and cerebellar beta stimulation $\left(t_{(23)}=2.91, p=0.01\right.$, $d=0.57$; Extended Data Fig. 4-1).

Control analyses indicated that recognition discrimination accuracy did not vary by session order regardless of stimulation condition (all pairwise $p$ values $>0.14$ ). Thus, session-to-session practice or ordering effects did not significantly contribute to the influence of stimulation on memory. Furthermore, there was no significant difference in accuracy for subjects receiving beta versus theta stimulation for the control-location condition $\left(t_{(22)}=0.79, p=0.46\right)$, indicating that rhythm-specific stimulation effects were unique to the cerebellum.

We performed two further analyses to support the assumption that these stimulation effects were relatively specific to episodic memory encoding (as the encoding period immediately followed stimulation and the recognition test was administered at a delay that is typically considered to be past the duration of stimulation effects on cognition for the current parameters; Rossi et al., 2009; Hoogendam et al., 2010; Thut and PascualLeone, 2010). If the effects of stimulation persisted during the recognition test, they would be expected to decay over the course of the recognition test, leading to variable performance between early and late memory testing, which might vary as a function of 

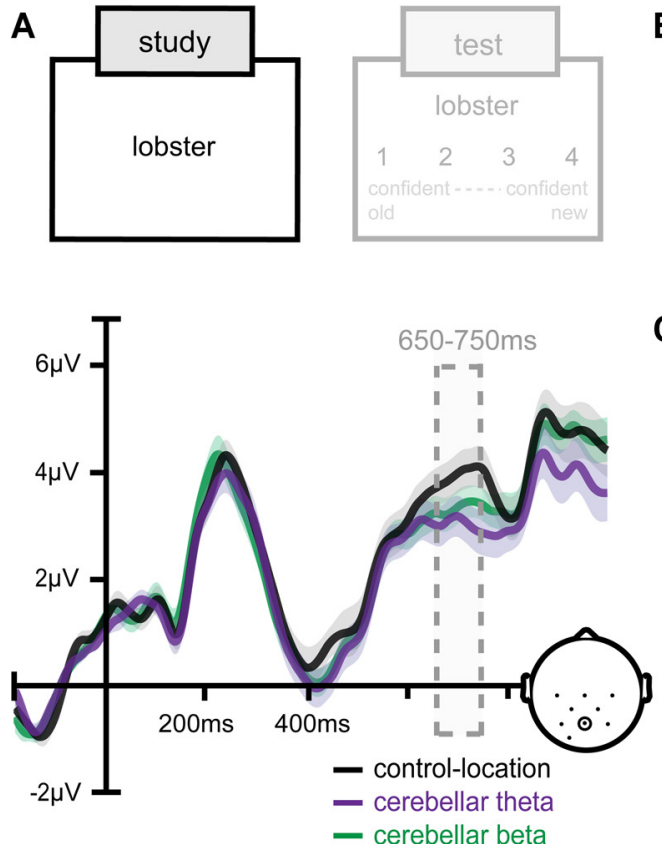

B
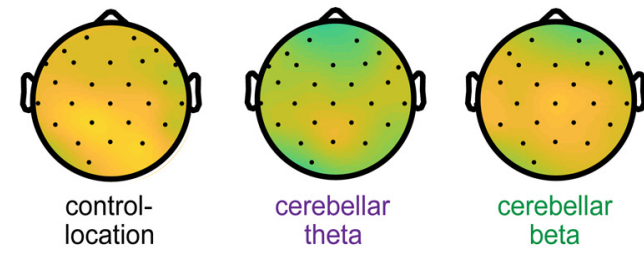

$-5 \mu \mathrm{V}$

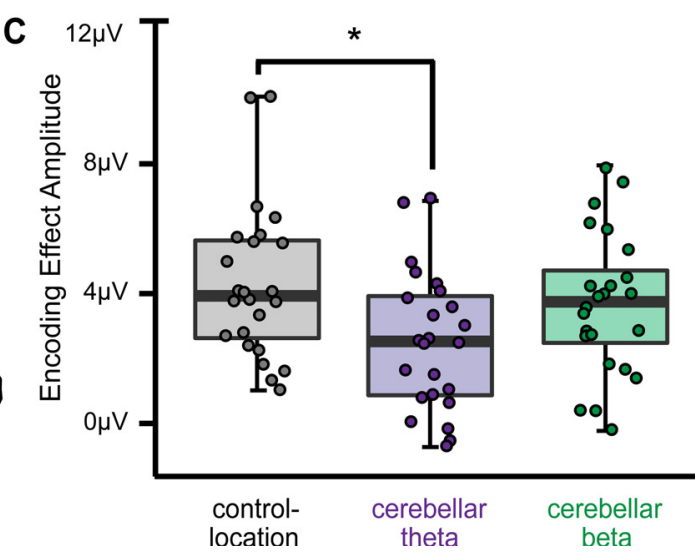

Figure 5. Cerebellar theta stimulation effects on ERP correlates of memory encoding. $A$, Average ERPs during single-word presentation are plotted for a representative centroposterior electrode (Pz). Encoding effect amplitudes $(650-750 \mathrm{~ms})$ differed by stimulation condition, with cerebellar theta stimulation reducing amplitudes relative to control-location stimulation. $\boldsymbol{B}$, Scalp topography of the parietal memory effect for each stimulation condition shows a greater, more widespread positive distribution following control-location stimulation relative to cerebellar theta-burst stimulation. C, Box-and-whisker plots of mean amplitudes between 650 and $750 \mathrm{~ms}$ per stimulation condition. Whiskers indicate first and third quartiles. Dots indicate values for individual subjects. Shading on ERP waveforms indicates SD of the group mean. ${ }^{*} p<0.05$.

stimulation condition. We therefore tested whether theta versus beta stimulation conditions differentially influenced performance for the first and last third of memory test trials ( $\sim 68-75$ vs 81-88 min after stimulation, respectively). Confident recognition discrimination accuracy did not vary between early and late memory trials for either stimulation condition (cerebellar beta stimulation: $t_{(23)}=0.83, p=0.41$; cerebellar theta stimulation: $\left.t_{(23)}=0.29, p=0.77\right)$, with no differential early versus late difference by condition $\left(t_{(23)}=0.45, p=0.66\right)$. Thus, if stimulation did continue to impact performance during the memory test, it did so throughout the memory test and to a similar extent for both stimulation conditions. This finding supports the conclusion that the impact of cerebellar theta stimulation on memory reflected an influence on encoding.

Next, we analyzed effects of stimulation on ERPs measured during single-word encoding, as an influence of cerebellar stimulation on these ERPs would further suggest an impact of theta stimulation on encoding. We focused on late-positive ERP amplitudes, as this signal has been associated with memory formation in many experiments (Rugg and Yonelinas, 2003; Voss and Paller, 2007). To ensure roughly equal trials across stimulation conditions, we analyzed late-positive ERP amplitude for all single-word encoding trials regardless of subsequent recognition performance (Fig. 5). Amplitudes were weakly modulated by stimulation condition $\left(F_{(2,46)}=2.96, p=0.06, \eta_{p}{ }^{2}=0.13\right)$, reflecting relatively lower late-positive amplitude following cerebellar theta stimulation versus location control stimulation $\left(t_{(46)}=2.06\right.$, $p=0.05, d=0.56$ ). ERP amplitudes following cerebellar beta stimulation did not significantly differ from either cerebellar theta $\left(t_{(46)}=1.20, p=0.24\right)$ or location control stimulation $\left(t_{(46)}=1.60, p=0.12\right)$. Thus, cerebellar theta stimulation weakly influenced ERP signals typical of word encoding when measured across all trials. Control analyses indicated that session order did not influence late-positive ERP amplitudes (all pairwise $p$ values $>0.12$ ). Additionally, subjects receiving beta versus theta stimulation of the control location did not have significantly different ERP amplitudes $\left(t_{(22)}=1.84, p=0.08\right)$. These results indicate that stimulation effects on ERP signals associated with encoding were specific to theta delivered to the cerebellum even when calculated on all words in aggregate, without considering trial-specific memory outcomes.

\section{Effects of stimulation on semantic prediction versus episodic memory}

Beta versus theta cerebellar stimulation had opposite effects on semantic prediction versus episodic memory. We quantified this pattern for the primary outcomes of semantic prediction versus episodic memory by analyzing the effects of stimulation frequency on normalized (z-scored) N400 amplitudes and recognition accuracy scores, computed relative to control-location stimulation. There was significant crossover interaction of stimulation frequency (theta vs beta) with functional outcome (normalized N400 vs normalized recognition accuracy; $F_{(1,23)}=7.02$, $p=0.01, \eta_{p}^{2}=0.29$ ), indicating cerebellar stimulation frequency had opposite effects on episodic memory versus semantic prediction.

This pattern of differential effects of beta versus theta stimulation can also be appreciated by considering standardized effect sizes from the ERP analyses that were performed separately for effects on semantic prediction and memory. Compared with the control-location stimulation condition, cerebellar beta stimulation had a larger impact on the N400 signal of semantic prediction than theta stimulation, whereas the opposite pattern (greater impact of theta than beta) was identified in late-positive ERPs during encoding (Fig. 6). Collectively, these findings support the conclusion that semantic prediction and memory were doubly 
dissociated based on different effects of beta versus theta cerebellar stimulation, respectively.

\section{Discussion}

Cerebellar beta stimulation increased N400 correlates of semantic prediction, suggesting enhanced discrimination of contextual predictability. In contrast, cerebellar theta stimulation affected ERP correlates of single-word encoding and led to improved memory accuracy. Our results indicate cerebellar beta and theta stimulation had differential effects on cognitive functions by stimulation rhythms, following the logic of the neuropsychological double dissociation (Teuber, 1955). The finding that cerebellar involvement in episodic memory and semantic prediction is dissociable under the command of rhythmic stimulation provides support for theories suggesting that the cerebellum coordinates with distributed networks via interregional synchronicity of oscillatory activity (Mantini et al., 2007; Uhlhaas et al., 2009). Thus, our findings of rhythm-specific effects of brain stimulation on distinct cognitive outcomes are consistent with this "communication-bysynchrony" hypothesis.

The distributed network associated with the hippocampus is important for episodic memory (Mesulam, 1990; Eichenbaum et al., 2007; Ranganath and Ritchey, 2012), and network interactions associated with memory have been correlated to synchronous activity in the theta band (Anderson et al., 2010; Lega et al., 2012). Resting-state fMRI connectivity is robust between the hippocampus and the ventrolateral cerebellum (Buckner et al., 2011), and theta-band phase synchrony occurs between the hippocampus and cerebellum during memory-related tasks (Wikgren et al., 2010). A previous experiment found that theta TMS targeting Crus I/II alters resting-state fMRI connectivity between the cerebellum and the hippocampal network as well as within the hippocampal network (Halko et al., 2014). Our results build on this finding by showing that theta stimulation was more effective than beta stimulation for memory enhancement. This finding is consistent with a previous study using parietal cortex TMS (Hermiller et al., 2019) and suggests that theta is relatively privileged in its ability to alter the function of the hippocampal network.

The N400 is a well characterized neural index of semantic processing, and thereby a very useful functional outcome of the frontotemporo-parietal language network (Kutas and Federmeier, 2011). N400 differences between highly predictable and less predictable words have been interpreted in the following two ways: as reflecting differences between the effort needed to contextually integrate an incoming word into an ongoing sentence, or as reflecting differences in how sentence context boosts prediction/preactivation of a specific word (Kuperberg and Jaeger, 2016). Although our experimental design is agnostic to this debate, it is noteworthy that the effects of cerebellar beta stimulation on the N400 were primarily driven by changes to the processing of low-predictability sentence endings. Stimulation may have influenced how words that were unlikely to be preactivated were processed (or, alternately, may have affected processing of words that require more effect to contextually integrate into the ongoing sentence). Notably, the effects of cerebellar stimulation on the N400 were specific to beta. In EEG and MEG studies examining oscillatory activity during sentences eliciting N400s, beta power was altered in response to contextual predictability (Wang et al., 2012; Molinaro et al., 2016). Whether these changes in beta are synchronous across regions in the frontal-temporal-parietal network that supports language comprehension and are active during predictive processing during reading (Lau et al., 2008; Hagoort, 2014) is still unknown. Nonetheless, our findings suggest

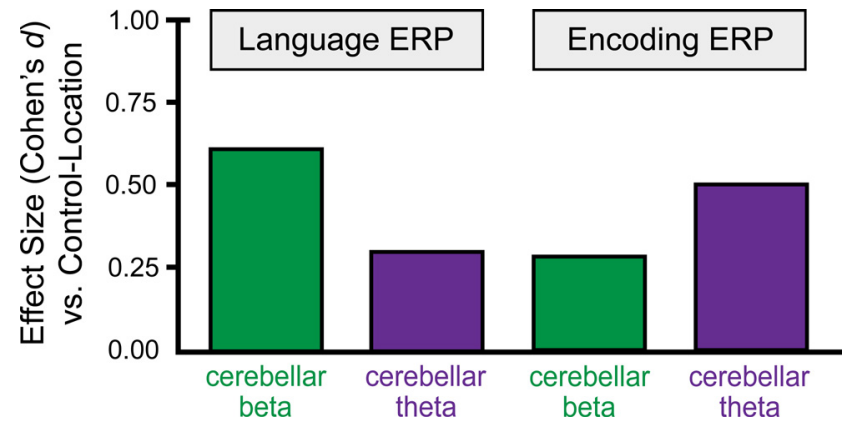

Figure 6. Opposite effects of stimulation rhythms on semantic prediction versus episodic memory. Standardized effect sizes for cerebellar theta and beta compared with control-location stimulation for N400 amplitudes and single-word encoding ERPs.

that beta is relatively privileged in its ability to alter the language network functions characterized by the N400.

The current "double dissociation" based on stimulation rhythm is notable with respect to the possibility of shared functional neuroanatomy for semantic prediction and episodic memory. Some have hypothesized theta-mediated hippocampal involvement in semantic prediction (Buckner, 2010; Bonhage et al., 2015; Davachi and DuBrow, 2015; Covington and Duff, 2016), perhaps because of the role of hippocampus in semantic memory (Piai et al., 2016) and/or in domain-general processing of prediction error (Covington and Duff, 2016; but see Ryskin et al., 2020). Nevertheless, we identified a double dissociation of semantic prediction and episodic memory based on cerebellar stimulation rhythm, suggesting functional distinction in terms of cognitive processing and relevant network mechanisms.

Our results are consistent with theories that brain stimulation impacts networks by entraining oscillatory activity (Buzsáki, 2002; Kim et al., 2016; Lea-Carnall et al., 2017). Importantly, we do not suggest that network activity remained entrained to the applied stimulation rhythm long after stimulation delivery, but rather that a lasting impact on network function was achieved by rhythm-matched stimulation because of an entrainment mechanism. In contrast to these resonance-oriented theories, compensation-oriented accounts propose that stimulation effects on local brain activity result in opposite effects in connected regions, via an unknown homeostatic mechanism (Eldaief et al., 2011; Cocchi et al., 2015). Continuous theta-burst stimulation has been characterized as locally inhibitory, whereas beta stimulation has been characterized as locally excitatory, based almost entirely on their effects when applied to primary motor cortex (PascualLeone et al., 1994; Di Lazzaro et al., 2005). Compensation-oriented theories therefore predict opposite effects of stimulation on cognitive processing (facilitation vs disruption). Our findings are inconsistent with this prediction, as stimulation frequencies led to different-but not opposite-levels of enhancement for different functions. Likewise, Hermiller et al. (2019) found that continuous theta-burst stimulation of a parietal cortex hub of the episodic memory network increased memory performance and memory-related fMRI connectivity to a greater degree than beta stimulation, but beta stimulation did not result in an opposite effect relative to sham stimulation. It has recently been demonstrated that stimulation parameters have nonuniform effects when applied to different cortical areas (Castrillon et al., 2020), calling into question the simple excitation/inhibition dichotomies derived from studies exclusive to primary motor cortex. 
Collectively, these results are consistent with resonance-oriented accounts of brain stimulation effects on cognition, whereby stimulation patterns matched to network-specific oscillations optimally facilitate network function. Nonetheless, the long-lasting impacts of effective rhythms are governed by as yet unknown mechanisms, which could include a variety of neuroplasticityrelated processes and are unlikely to reflect long-lasting resonance of neural activity following stimulation.

The lateral cerebellar cortex is heavily and highly regularly connected with cerebral association cortex, prompting speculation that Crus I may make similar functional contributions to multiple cognitive brain networks (Schmahmann, 2019). The internal model hypothesis (Ito, 2008) suggests that the cerebellum encodes internal models concerning the mental representations of incoming information that are held in the cerebral cortex. The cerebellar cortex then generates and updates (via feedback) internal models. Semantic prediction and memory encoding rely on feedback-related updating of internal models, which could be similarly provided by Crus I. Our findings suggest that this contribution may be selectively upregulated for semantic prediction versus memory via application of the distinct rhythms matching their corresponding functional networks.

One limitation of the current study is that mechanistic interpretations are limited by the nature of the episodic memory and language outcomes measured. There are compelling models for the relevance of theta-gamma coordination for episodic memory (Shirvalkar et al., 2010; Lisman and Jensen, 2013; Buzsáki and Schomburg, 2015) and of beta coordination with multiple frequencies for semantic prediction (Saleh et al., 2010; Arnal et al., 2015). Our noninvasive measurement was not able to determine whether stimulation altered circuit-specific timing of activity and cannot evaluate such mechanisms. However, the frequency-specific impact of stimulation on cognitive function is consistent with the idea that oscillatory activity was affected by cerebellar stimulation, at least temporarily. Further, although the experiment design and results suggest that the effect of cerebellar theta stimulation was on memory encoding, including control analyses that suggest no lingering differential impact of theta versus beta stimulation on retrieval, we did not also test for selective encoding effects by delivering stimulation between encoding and retrieval, and so cannot be entirely confident that only encoding was improved versus other stages of memory processing. Finally, although we targeted cerebellar Crus I, the induced electrical field could have influenced other areas of cerebellum, and our conclusions regarding the functional neuroanatomical specificity of the observed influences of stimulation are limited by the lack of localization data, such as provided by fMRI or related methods. These are issues that could be addressed in future experiments.

The current findings could be relevant to the use of noninvasive brain stimulation for the treatment of language and episodic memory impairments, such as those that occur because of neurodegeneration of corresponding brain networks (Golomb et al., 1993; Mesulam et al., 2014). Rhythm-specific stimulation effects on language versus episodic memory could provide a means to efficiently target specific symptoms in patient groups to achieve relatively personalized treatments. Furthermore, the cerebellum may be superior to cerebral cortical targets for stimulation in neurodegenerative disease because it is relatively less affected by neuropathological processes (Marcyniuk et al., 1986; Mann et al., 1990), although it is not immune to them (Guo et al., 2016; Schmahmann, 2016). Further investigation of rhythm-specific effects of cerebellar stimulation could thus advance understanding of cerebellar contributions to cognitive function while also motivating novel treatment approaches for cognitive impairment.

\section{References}

Anderson KL, Rajagovindan R, Ghacibeh GA, Meador KJ, Ding M (2010) Theta oscillations mediate interaction between prefrontal cortex and medial temporal lobe in human memory. Cereb Cortex 20:16041612.

Arnal LH, Doelling KB, Poeppel D (2015) Delta-beta coupled oscillations underlie temporal prediction accuracy. Cereb Cortex 25:3077-3085.

Bastiaansen M, Hagoort P (2006) Oscillatory neuronal dynamics during language comprehension. Prog Brain Res 159:179-196.

Bohbot VD, Copara MS, Gotman J, Ekstrom AD (2017) Low-frequency theta oscillations in the human hippocampus during real-world and virtual navigation. Nat Commun 8:14415.

Bonhage CE, Mueller JL, Friederici AD, Fiebach CJ (2015) Combined eye tracking and fMRI reveals neural basis of linguistic predictions during sentence comprehension. Cortex 68:33-47.

Brysbaert M, Warriner AB, Kuperman V (2014) Concreteness ratings for 40 thousand generally known English word lemmas. Behav Res Methods 46:904-911.

Buckner RL (2010) The role of the hippocampus in prediction and imagination. Annu Rev Psychol 61:27-48.

Buckner RL, Krienen FM, Castellanos A, Diaz JC, Yeo BT (2011) The organization of the human cerebellum estimated by intrinsic functional connectivity. J Neurophysiol 106:2322-2345.

Buzsáki G (2002) Theta oscillations in the hippocampus. Neuron 33:325340.

Buzsáki G, Schomburg EW (2015) What does gamma coherence tell us about inter-regional neural communication? Nat Neurosci 18:484-489.

Castrillon G, Sollmann N, Kurcyus K, Razi A, Krieg SM, Riedl V (2020) The physiological effects of noninvasive brain stimulation fundamentally differ across the human cortex. Sci Adv 6:eaay2739.

Cocchi L, Sale MV, Lord A, Zalesky A, Breakspear M, Mattingley JB (2015) Dissociable effects of local inhibitory and excitatory theta-burst stimulation on large-scale brain dynamics. J Neurophysiol 113:3375-3385.

Covington NV, Duff MC (2016) Expanding the language network: direct contributions from the hippocampus. Trends Cogn Sci 20:869-870.

Cox RW (1996) AFNI: software for analysis and visualization of functional magnetic resonance neuroimages. Computers and Biomedical research, $29: 162-173$

Davachi L, DuBrow S (2015) How the hippocampus preserves order: the role of prediction and context. Trends Cogn Sci 19:92-99.

Dave S, Brothers TA, Swaab TY (2018) 1/f neural noise and electrophysiological indices of contextual prediction in aging. Brain Res 1691:34-43.

De Smet HJ, Paquier P, Verhoeven J, Mariën P (2013) The cerebellum: its role in language and related cognitive and affective functions. Brain Lang 127:334-342.

Desmond JE, Fiez JA (1998) Neuroimaging studies of the cerebellum: language, learning and memory. Trends Cogn Sci 2:355-362.

Di Lazzaro V, Pilato F, Saturno E, Oliviero A, Dileone M, Mazzone P, Insola A, Tonali PA, Ranieri F, Huang YZ, Rothwell JC (2005) Theta-burst repetitive transcranial magnetic stimulation suppresses specific excitatory circuits in the human motor cortex. J Physiol 565:945-950.

Diana RA, Yonelinas AP, Ranganath C (2007) Imaging recollection and familiarity in the medial temporal lobe: a three-component model. Trends Cogn Sci 11:379-386.

Eichenbaum H, Yonelinas AP, Ranganath C (2007) The medial temporal lobe and recognition memory. Annu Rev Neurosci 30:123-152.

Eldaief MC, Halko MA, Buckner RL, Pascual-Leone A (2011) Transcranial magnetic stimulation modulates the brain's intrinsic activity in a frequency-dependent manner. Proc Natl Acad Sci U S A 108:2122921234.

Engel AK, Fries P, Singer W (2001) Dynamic predictions: oscillations and synchrony in top-down processing. Nat Rev Neurosci 2:704-716.

Fliessbach K, Trautner P, Quesada CM, Elger CE, Weber B (2007) Cerebellar contributions to episodic memory encoding as revealed by fMRI. Neuroimage 35:1330-1337. 
Golomb J, de Leon MJ, Kluger A, George AE, Tarshish C, Ferris SH (1993) Hippocampal atrophy in normal aging. An association with recent memory impairment. Arch Neurol 50:967-973.

Guo CC, Tan R, Hodges JR, Hu X, Sami S, Hornberger M (2016) Networkselective vulnerability of the human cerebellum to Alzheimer's disease and frontotemporal dementia. Brain 139:1527-1538.

Hagoort P (2014) Nodes and networks in the neural architecture for language: Broca's region and beyond. Curr Opin Neurobiol 28:136-141.

Halko MA, Farzan F, Eldaief MC, Schmahmann JD, Pascual-Leone A (2014) Intermittent theta-burst stimulation of the lateral cerebellum increases functional connectivity of the default network. J Neurosci 34:1204912056.

Hermiller MS, VanHaerents S, Raij T, Voss JL (2019) Frequency-specific noninvasive modulation of memory retrieval and its relationship with hippocampal network connectivity. Hippocampus 29:595-609.

Herweg NA, Solomon EA, Kahana MJ (2020) Theta oscillations in human memory. Trends Cogn Sci 24:208-227.

Holcomb PJ, Grainger J (2006) On the time course of visual word recognition: an event-related potential investigation using masked repetition priming. J Cogn Neurosci 18:1631-1643.

Hoogendam JM, Ramakers GMJ, Di Lazzaro V (2010) Physiology of repetitive transcranial magnetic stimulation of the human brain. Brain Stimul 3:95-118.

Huang YY, Kandel ER (2005) Theta frequency stimulation induces a local form of late phase LTP in the CA1 region of the hippocampus. Learn Mem 12:587-593.

Ito M (2008) Control of mental activities by internal models in the cerebellum. Nat Rev Neurosci 9:304-313.

Jacoby LL (1983) Perceptual enhancement: persistent effects of an experience. J Exp Psychol Learn Mem Cogn 9:21-38.

Janssen AM, Oostendorp TF, Stegeman DF (2015) The coil orientation dependency of the electric field induced by TMS for M1 and other brain areas. J Neuroeng Rehabil 12:47.

Kim K, Ekstrom AD, Tandon N (2016) A network approach for modulating memory processes via direct and indirect brain stimulation: toward a causal approach for the neural basis of memory. Neurobiol Learn Mem 134:162-177.

King M, Hernandez-Castillo CR, Poldrack RA, Ivry RB, Diedrichsen J (2019) Functional boundaries in the human cerebellum revealed by a multi-domain task battery. Nat Neurosci 22:1371-1378.

Krienen FM, Buckner RL (2009) Segregated fronto-cerebellar circuits revealed by intrinsic functional connectivity. Cereb Cortex 19:24852497.

Kuperberg GR, Jaeger TF (2016) What do we mean by prediction in language comprehension? Lang Cogn Neurosci 31:32-59.

Kutas M, Federmeier KD (2011) Thirty years and counting: finding meaning in the N400 component of the event-related brain potential (ERP). Annu Rev Psychol 62:621-647.

Larson J, Wong D, Lynch G (1986) Patterned stimulation at the theta frequency is optimal for the induction of hippocampal long-term potentiation. Brain Res 368:347-350.

Lau EF, Phillips C, Poeppel D (2008) A cortical network for semantics: (de) constructing the N400. Nat Rev Neurosci 9:920-933.

Lea-Carnall CA, Trujillo-Barreto NJ, Montemurro MA, El-Deredy W, Parkes LM (2017) Evidence for frequency-dependent cortical plasticity in the human brain. Proc Natl Acad Sci U S A 114:8871-8876.

Leckey M, Federmeier KD (2020) The P3b and P600(s): positive contributions to language comprehension. Psychophysiology 57:e13351.

Lega BC, Jacobs J, Kahana M (2012) Human hippocampal theta oscillations and the formation of episodic memories. Hippocampus 22:748-761.

Lewis AG, Bastiaansen M (2015) A predictive coding framework for rapid neural dynamics during sentence-level language comprehension. Cortex 68:155-168.

Lisman JE, Jensen O (2013) The theta-gamma neural code. Neuron 77:10021016.

Lopez-Calderon J, Luck SJ (2014) ERPLAB: an open-source toolbox for the analysis of event-related potentials. Front Hum Neurosci 8:213.

Mann DM, Jones D, Prinja D, Purkiss MS (1990) The prevalence of amyloid (A4) protein deposits within the cerebral and cerebellar cortex in Down's syndrome and Alzheimer's disease. Acta Neuropathol 80:318327.
Mantini D, Perrucci MG, Del Gratta C, Romani GL, Corbetta M (2007) Electrophysiological signatures of resting state networks in the human brain. Proc Natl Acad Sci U S A 104:13170-13175.

Marcyniuk B, Mann DM, Yates PO (1986) The topography of cell loss from locus caeruleus in Alzheimer's disease. J Neurol Sci 76:335-345.

Mesulam MM (1990) Large-scale neurocognitive networks and distributed processing for attention, language, and memory. Ann Neurol 28:597613.

Mesulam M-M, Rogalski EJ, Wieneke C, Hurley RS, Geula C, Bigio EH, Thompson CK, Weintraub S (2014) Primary progressive aphasia and the evolving neurology of the language network. Nat Rev Neurol 10:554-569.

Molinaro N, Monsalve IF, Lizarazu M (2016) Is there a common oscillatory brain mechanism for producing and predicting language? Lang Cogn Neurosci 31:145-158.

Mottaghy FM, Hungs M, Brugmann M, Sparing R, Boroojerdi B, Foltys H, Huber W, Topper R (1999) Facilitation of picture naming after repetitive transcranial magnetic stimulation. Neurology 53:1806-1812.

Pascual-Leone A, Valls-Solé J, Wassermann EM, Hallett M (1994) Responses to rapid-rate transcranial magnetic stimulation of the human motor cortex. Brain 117:847-858

Piai V, Anderson KL, Lin JJ, Dewar C, Parvizi J, Dronkers NF, Knight RT (2016) Direct brain recordings reveal hippocampal rhythm underpinnings of language processing. Proc Natl Acad Sci U S A 113:1136611371

Ranganath C, Ritchey M (2012) Two cortical systems for memory-guided behaviour. Nat Rev Neurosci 13:713-726.

Rossi S, Hallett M, Rossini PM, Pascual-Leone A (2009) Safety, ethical considerations, and application guidelines for the use of transcranial magnetic stimulation in clinical practice and research. Clin Neurophysiol 120:2008-2039.

Rugg MD, Yonelinas AP (2003) Human recognition memory: a cognitive neuroscience perspective. Trends Cogn Sci 7:313-319.

Ryskin R, Levy RP, Fedorenko E (2020) Do domain-general executive resources play a role in linguistic prediction? Re-evaluation of the evidence and a path forward. Neuropsychologia 136:107258.

Saleh M, Reimer J, Penn R, Ojakangas CL, Hatsopoulos NG (2010) Fast and slow oscillations in human primary motor cortex predict oncoming behaviorally relevant cues. Neuron 65:461-471.

Schmahmann JD (2016) Cerebellum in Alzheimer's disease and frontotemporal dementia: not a silent bystander. Brain 139:1314-1318.

Schmahmann JD (2019) The cerebellum and cognition. Neurosci Lett 688:62-75.

Shain C, Blank IA, van Schijndel M, Schuler W, Fedorenko E (2020) fMRI reveals language-specific predictive coding during naturalistic sentence comprehension. Neuropsychologia 138:107307.

Shepard RN (1967) Recognition memory for words, sentences, and pictures. J Verbal Learning Verbal Behav 6:156-163.

Shirvalkar PR, Rapp PR, Shapiro ML (2010) Bidirectional changes to hippocampal theta-gamma comodulation predict memory for recent spatial episodes. Proc Natl Acad Sci U S A 107:7054-7059.

Sparing R, Mottaghy FM, Hungs M, Brügmann M, Foltys H, Huber W, Töpper R (2001) Repetitive transcranial magnetic stimulation effects on language function depend on the stimulation parameters. J Clin Neurophysiol 18:326-330.

Stoodley CJ (2012) The cerebellum and cognition: evidence from functional imaging studies. Cerebellum 11:352-365.

Teuber HL (1955) Physiological psychology. Annu Rev Psychol 6:267-296.

Thielscher A, Antunes A, Saturnino GB (2015) Field modeling for transcranial magnetic stimulation: a useful tool to understand the physiological effects of TMS? Conf Proc IEEE Eng Med Biol Soc 2015:222225.

Thut G, Pascual-Leone A (2010) A review of combined TMS-EEG studies to characterize lasting effects of repetitive TMS and assess their usefulness in cognitive and clinical neuroscience. Brain Topogr 22:219-232.

Uhlhaas PJ, Roux F, Singer W, Haenschel C, Sireteanu R, Rodriguez E (2009) The development of neural synchrony reflects late maturation and restructuring of functional networks in humans. Proc Natl Acad Sci U S A 106:9866-9871.

Van Petten C, Luka BJ (2012) Prediction during language comprehension: benefits, costs, and ERP components. Int J Psychophysiol 83:176-190. 
Voss JL, Paller KA (2007) Neural correlates of conceptual implicit memory and their contamination of putative neural correlates of explicit memory. Learn Mem 14:259-267.

Wang L, Jensen $\mathrm{O}$, van den Brink $\mathrm{D}$, Weder N, Schoffelen J-M, Magyari L, Hagoort P, Bastiaansen M (2012) Beta oscillations relate to the N400m during language comprehension. Hum Brain Mapp 33:2898-2912.

Weiss S, Mueller HM (2012) "Too many betas do not spoil the broth": the role of beta brain oscillations in language processing. Front Psychol 3:201.

Wikgren J, Nokia MS, Penttonen M (2010) Hippocampo-cerebellar theta band phase synchrony in rabbits. Neuroscience 165:1538-1545.
Yeo BTT, Krienen FM, Sepulcre J, Sabuncu MR, Lashkari D, Hollinshead M, Roffman JL, Smoller JW, Zöllei L, Polimeni JR, Fischl B, Liu H, Buckner RL (2011) The organization of the human cerebral cortex estimated by intrinsic functional connectivity. J Neurophysiol 106:1125-1165.

Yonelinas AP (1999) The contribution of recollection and familiarity to recognition and source-memory judgments: a formal dual-process model and an analysis of receiver operating characteristics. J Exp Psychol Learn Mem Cogn 25:1415-1434.

Yonelinas AP (2002) The nature of recollection and familiarity: a review of 30 years of research. J Mem Lang 46:441-517.

Zhang H, Jacobs J (2015) Traveling theta waves in the human hippocampus. J Neurosci 35:12477-12487. 